\title{
Déconstruire en exposant
}

Ernst Jünger, travailleur au bord de l'abîme aux Archives littéraires allemandes de Marbach

\section{Heike Gfrereis}

Traducteur : Aude Therstappen

\section{OpenEdition Journals}

\section{Édition électronique}

URL : https://journals.openedition.org/rbnu/3454

DOI : $10.4000 /$ rbnu.3454

ISSN : 2679-6104

\section{Éditeur}

Bibliothèque nationale et universitaire de Strasbourg

\section{Édition imprimée}

Date de publication : 1 mai 2011

Pagination : 40-47

ISSN : 2109-2761

\section{Référence électronique}

Heike Gfrereis, « Déconstruire en exposant », La Revue de la BNU [En ligne], 3 | 2011, mis en ligne le 01 mai 2011, consulté le 08 août 2021. URL : http://journals.openedition.org/rbnu/3454 ; DOI : https:// doi.org/10.4000/rbnu.3454

\section{(c) $)(1)(2)$}

La Revue de la BNU est mise à disposition selon les termes de la Licence Creative Commons Attribution - Pas d'Utilisation Commerciale - Partage dans les Mêmes Conditions 4.0 International. 
I.

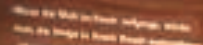

\section{ancos}

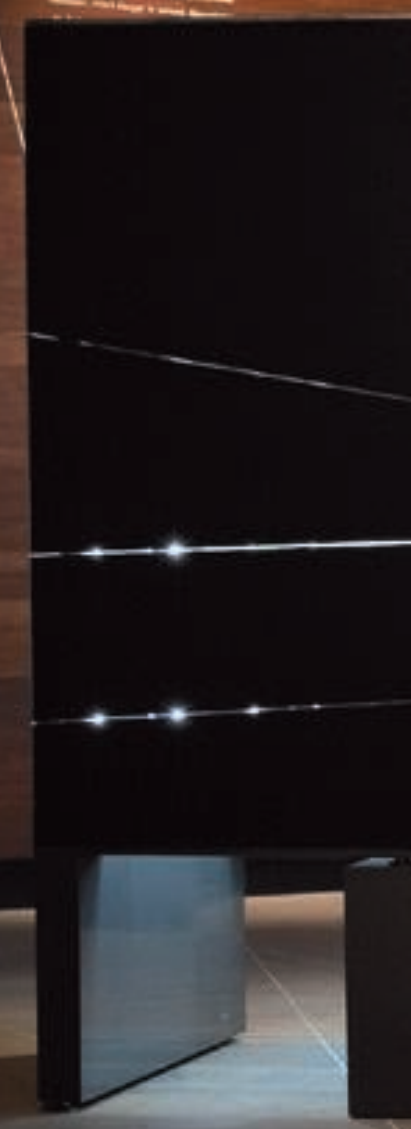

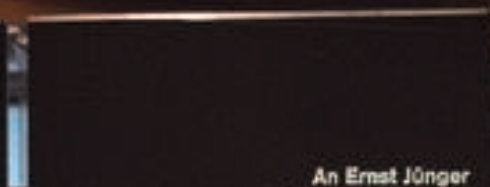
schelden sich immer. noch dila Galster.

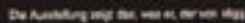

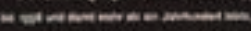

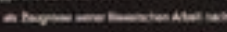
nowesenes
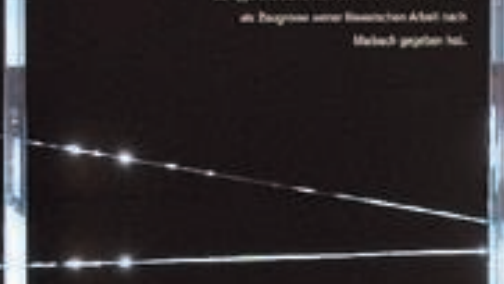

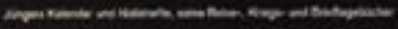

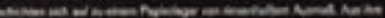

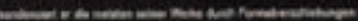

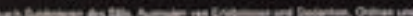

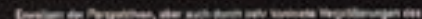

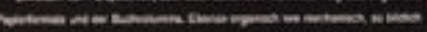

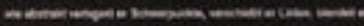

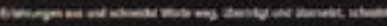

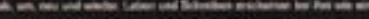

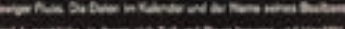

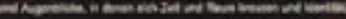
compunas

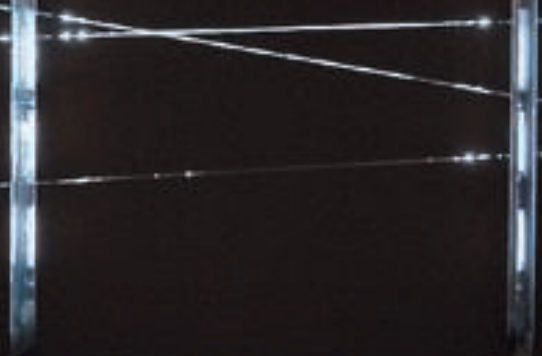

t:

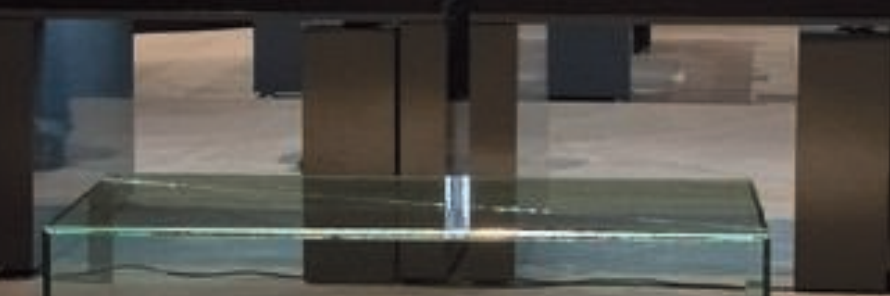




\section{Dé-construire en exposant : Ernst Jünger, travailleur au bord de l'abîme aux Archives littéraires allemandes de Marbach}

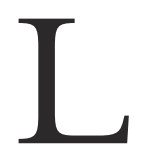

es Archives littéraires allemandes de Marbach ont acquis le fonds Ernst Jünger en 1994, un an avant son centième anniversaire et quatre ans avant sa mort. Ce fonds est riche de très nombreuses versions de manuscrits et de tapuscrits des textes de Jünger et de ses œuvres complètes, mais aussi de pas moins de 252 calendriers et journaux intimes encore inédits, de plus de 30 ooo lettres et d'un grand nombre de photos, sans oublier la bibliothèque de l'écrivain et sa collection d'objets d'art qui demeurent toutefois dans sa maison de Wilflingen (officiellement classée monument historique en 1999) pour en préserver l'aménagement d'origine. L'exposition Ernst Jünger, travailleur au bord de l'abîme (qui s'est tenue du 7 novembre 2010 au 27 mars 2011 au Musée littéraire de la modernité) a montré ce fonds pour la première fois au public. A travers cet exemple, je voudrais présenter les pratiques d'expositions qui sont les nôtres à Marbach, ainsi que l'arrière-plan théorique dont elles découlent.

\section{Penser avec les choses dans l'espace}

Depuis l'ouverture, en 2006, du Musée littéraire de la modernité (Literaturmuseum der Moderne), conçu par l'architecte David Chipperfield, les Archives disposent d'une surface de $400 \mathrm{~m}^{2}$ dévolue aux expositions temporaires, en plus des deux expositions permanentes au Musée littéraire et au
Musée Schiller. Il est important de trouver, pour ces expositions temporaires, des thèmes qui soient d'une part susceptibles de constituer un matériau neuf et inexploité, pas encore rangé et classifié par les chercheurs, et qui d'autre part donnent de nouvelles impulsions à la recherche. Nous ne devons pas nous contenter de traduire ces impulsions en images mises en espace mais il faut au contraire s'efforcer, à travers une certaine disposition et une certaine réflexion, de permettre que ce travail de recherche naisse en quelque sorte de notre présentation. Faire des expositions, cela signifie pour nous trouver ce qui, dans les fonds des archives, ne peut être perçu que grâce à une organisation mise en espace et l'exposer de telle sorte que chaque visiteur, qu'il s'agisse ou non d'un spécialiste, puisse faire ses propres découvertes à partir du moment où il accepte de se laisser embarquer dans l'exposition. Pour chacune des grandes expositions réalisées depuis 2006, nous avons toujours trouvé, voire inventé, une façon originale de montrer les documents. Nous avons tour à tour accroché, couché, laissé droit, aligné, entassé, mis en croix, accumulé, combiné, bougé, compacté ou bien encore isolé les pièces pour suivre à chaque fois une logique du visible, du temps, de l'espace, du genre, de l'œuvre ou du corps.

Nous avons toujours fait en sorte que notre travail de sélection et d'ordonnancement soit clair, afin que les visiteurs puissent adopter notre point de vue et ne soient pas confrontés à des conclusions toutes faites. L'évidence est quelque chose qui naît dans l'esprit 
$\mathrm{du}$ spectateur. Nous pouvons mettre en place les conditions qui permettent la découverte mais nous ne pouvons l'imposer et c'est pourquoi nous veillons à ce que les expositions conservent une certaine distance, à ce que les documents et les explications soient bien séparés. Nous séparons également nettement les différents médias et renonçons à la synesthésie et à la proximité. Le matériau exposé devient " brûlant " à partir du moment où le visiteur regarde attentivement ces systèmes étrangers et non leur mise en espace.

\section{La ligne de l'écriture}

La réception allemande de Jünger a été et reste encore dominée par deux variantes de lecture problématiques : la condamnation morale ou la défense de l'auteur, deux attitudes qui confortent avant tout le lecteur dans ses propres convictions. Nous nous sommes donc concentrés sur ce qui, dans le fonds Jünger, témoigne de son travail d'écrivain et a dominé une grande partie de sa longue existence : les textes plus que la vie, les mots plus que les actes, les prises de position esthétiques plus que les opinions idéologiques. L'exposition utilise deux méthodes et techniques heuristiques : l'alignement sériel et antithétique, et la combinaison associative et métaphorique. Une grande vitrine suit la chronologie du travail d'écriture de Jünger et montre le processus de création littéraire, de systématisation, de codage, formatage et réécriture de ses journaux intimes. La vitrine représente la ligne " magique ", ce seuil qui relie constamment les journaux intimes à l'œuvre et qui constitue un plan horizontal que les autres vitrines basses viennent couper. Les journaux intimes placés en haut sont mis en parallèle des œuvres exposées au-dessous. Les lacunes dans la documentation sont représentées par des vides dans l'exposition, comme pour les années vingt, époque pour laquelle Jünger a détruit presque tous ses papiers après une fouille de la Gestapo, ou encore pour mai et juin 1944 où il a arraché les pages de ses calendriers. La structure auto-poétique de cette écriture, dans laquelle il n'y a qu'une seule logique et une seule réalité, celle du texte et de son sujet au détriment de celle de la vie et de l'homme, cette structure devient visible. Nous donnons à voir des stratégies esthétiques, non des formes de réécriture et de traduction ; des procédés de construction d'un texte à partir d'une abondance de matériel et de versions, non les étapes de la genèse textuelle depuis l'idée jusqu'à l'œuvre finie. Pour Jünger, l'œuvre n'est pas liée à une forme atemporelle, à un style éternel et parfait mais bien à un instant et à un espace. Est œuvre ce qui existe ici et maintenant et sera transposé demain peut-être dans une autre version ou bien encore détruit. Les visiteurs peuvent se faire une idée d'ensemble de cette technique de travail particulière en découvrant les journaux intimes de Jünger du premier au dernier. Ils peuvent suivre la façon dont les dates, les mots, les images des journaux intimes trouvent leur place dans les œuvres de grand format des vitrines inférieures. De nouveaux rapports entre les choses s'organisent et les visiteurs peuvent se faire une première idée, au plus près possible des manuscrits originaux et aussi facilement et rapidement que possible, du travail d'écriture de Jünger, travail d'une vie entière et de près de quatrevingt-dix années. Tenir son journal intime était pour Jünger un art de vivre bien que, dans le journal original, la vie paraisse parfois paradoxalement plus symbolique et fictive que dans le texte publié. "Il faut voir à quel point Jünger remplit des carnets et des calendriers non seulement d'écriture mais aussi d'esquisses, d'éléments décoratifs et de tableaux. Ainsi, on peut admirer toute une culture de l'illustration qui prolifère dans les textes, un syncrétisme des formats et des styles et justement pas cette dureté métallique du matériau qu'exige un certain dandysme littéraire " (Frankfurter Allgemeine Zeitung, Daniel Haas).

\section{Sur le mur du temps}

Dix chapitres thématiques sont disposés à côté de la " colonne vertébrale " chronologique qui occupe les deux salles de l'exposition. Ces chapitres s'appuient sur les constantes centrales de l'écriture de Jünger, c'est-à-dire sur leurs phénomènes les plus évidents qui, jusque dans les métaphores les plus fréquemment utilisées, conduisent à comprendre ses positions, pensées, écrites et vécues mais aussi esthétiques, philosophiques et politiques. Dans ces chapitres, nous avons combiné différentes sortes d'objets et présenté certaines parties choisies des collections de Wilflingen, car ces objets, souvent décrits dans son œuvre (comme les célèbres casques d'acier, les " armes optiques " sur son bureau ou encore les coléoptères des " chasses subtiles "), possèdent en eux-mêmes ou 
"Ici, chaque classique est examiné jusqu'à l'os comme lors d'une radiographie. Il ne reste pas grand-chose de la grandeur marmoréenne.

Il n'en a pas été autrement avec Jünger. Ernst Jünger, travailleur au bord de l'abîme donne la mesure de ce qu'une exposition littéraire peut apporter à la compréhension d'une œuvre ".

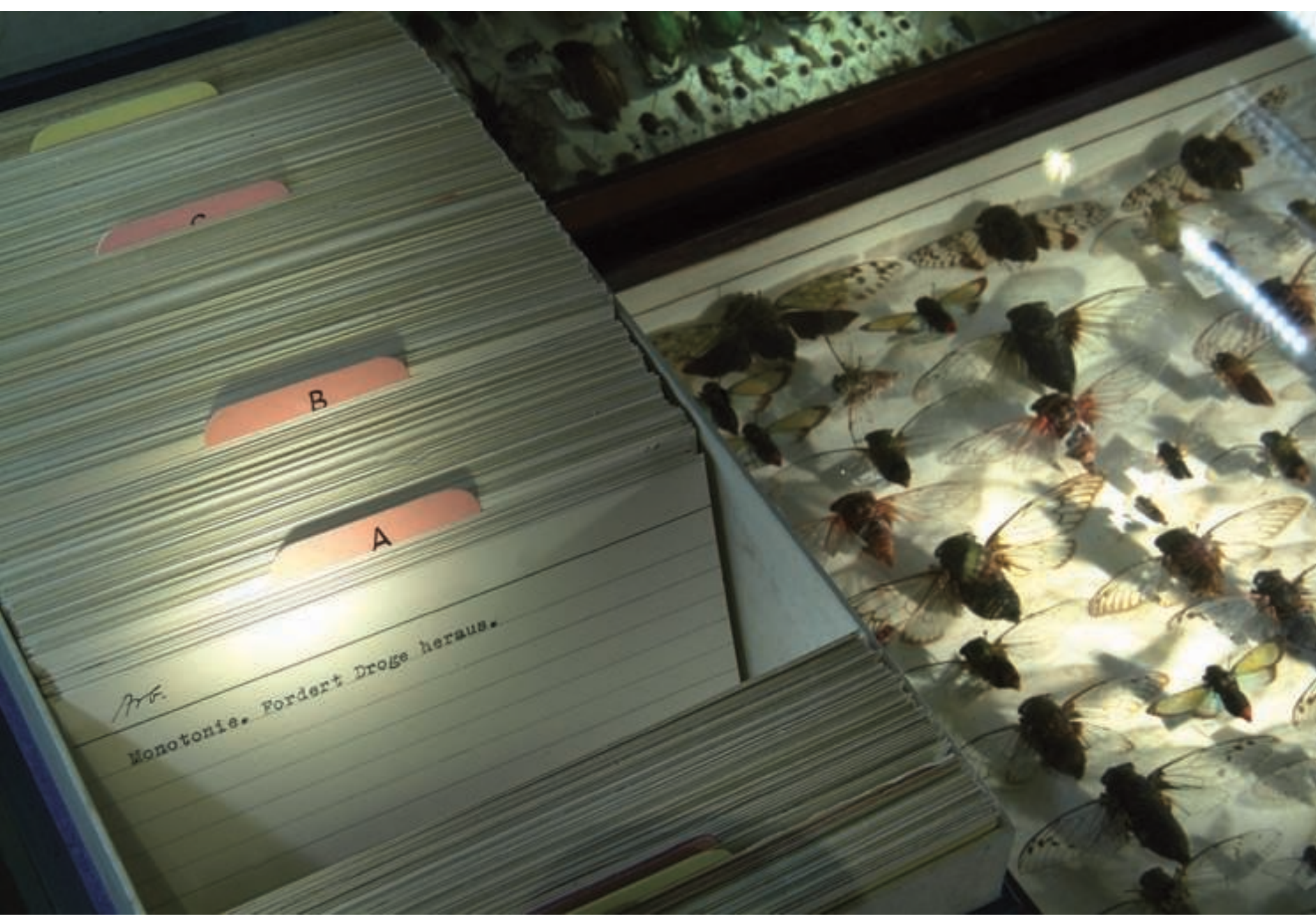




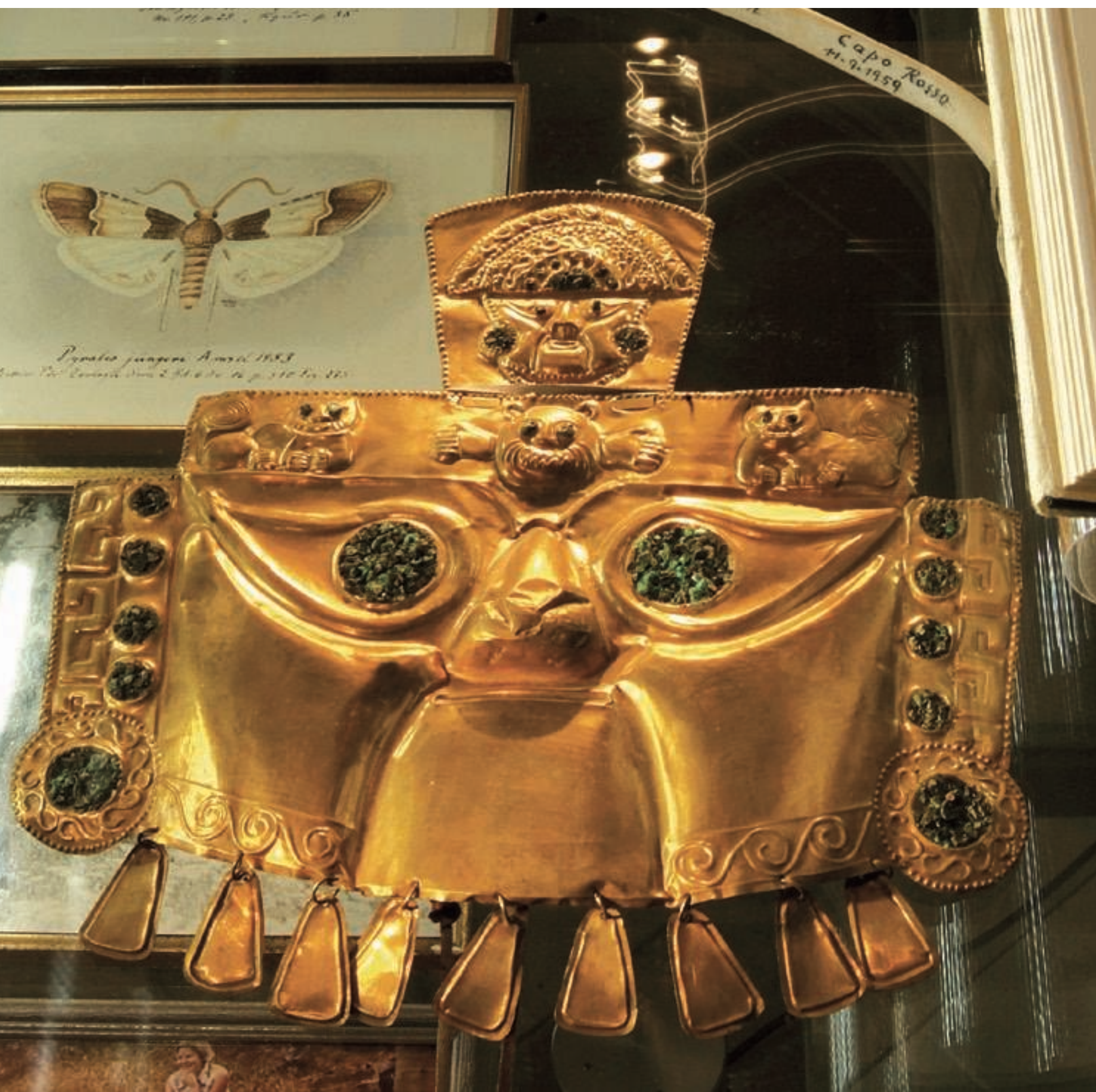




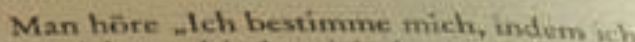
untergiehe und indem ich derari mich sell tonch me eine W elt" usw. Ich mich mir selbst. Hai uns besionk. ten davor geekelt? Sind wir dem ehrluchen to davon gelaufen, um diese dreisten lch V Vrrithat. zumachen? Aber weil es in Deutschland unusei es hier erlaube und keiner wagr zu lachen. Ir Buribunke, wie er im Buch des Existeriatisimen nur franzosisch zu sprechen und er hat alle selbst. Ich bin Ich nur leh allein, rief mein Nar 1845. Das also steht heute unter Besathumgsse das Römische Recht unter Achsensehutr. F

1. Am Rande von C. S, offenbar spăter vetmer Phantasie, 1. Fliegel: der Wasserunhold vtirbe un a Wallfahrsort im fre. Département Tsere, Celle qui pleure (Notre Darne de la Saleur). (Nachdruck 1949) behandelt hat.

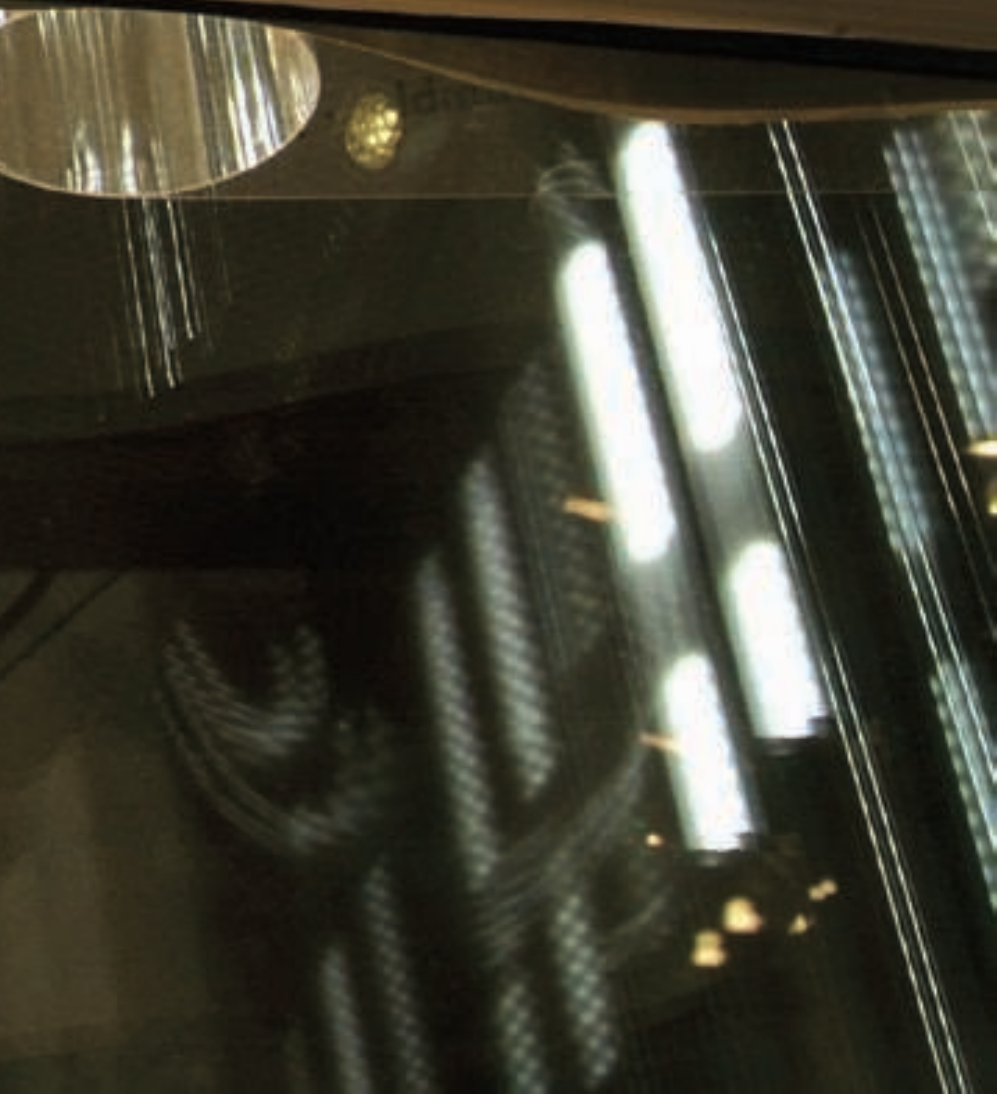

par la façon dont ils y sont disposés des particularités analogues à celles des procédés stylistiques utilisés par l'auteur.

En ce qui concerne la colonne vertébrale de l'exposition, l'infatigable travail de réécriture de Jünger, les critères de sélection étaient clairs (tous les journaux intimes et tous les manuscrits principaux correspondants, ou encore, lorsqu'ils ne sont pas conservés comme dans le cas d'Orages d'acier, des livres issus du fonds Jünger). En revanche, les critères de sélection des objets thématiques étaient subjectifs : nous avons exposé des objets qui nous paraissaient être en relation esthétique avec l'œuvre de Jünger ou que son œuvre littéraire rendait intelligibles, comme dans le cas de la stéréoscopie ou de ses nombreuses publications nationalistes écrites dans les années vingt. Il n'était pas question d'en gommer l'aspect dangereux et explosif. Les textes de Jünger sont ambigus comme le sont ces objets exposés. Les métaphores de son style fleuri sont aussi souvent d'origine militaire que biblique et rappellent aussi bien la création que la destruction, qu'il connote d'ailleurs toutes deux positivement. Jünger n'est pas un écrivain des lamentations ni de la consternation. Ce que les pièces exposées ne font pas, c'est illustrer, instruire, raconter, documenter ou révéler des secrets. Elles montrent ce que les lecteurs de Jünger apprécient et qui n'est pas pour rien dans la fascination que ses textes provoquent en eux : l'accumulation de pensées et de faits chargés d'une signification difficile à définir, libre car ne pouvant être utilisée ou détournée.

\section{Du côté des pièces exposées}

L'exposition permanente du Musée littéraire de la modernité sépare de façon radicale le niveau de l'illustration et le niveau de la transmission. Les visiteurs trouvent toutes les informations sur un ordinateur portable. Dans les expositions temporaires aussi, nous jouons sciemment avec les genres et les masses de textes. Parfois on n'a besoin d'aucun accompagnement explicatif (quand on peut tout simplement montrer l'objet comme dans le cas des carnets de Robert Gernhardt, qu'il a remplis de 1978 jusqu'à sa mort de croquis et de notes et que nous avons accrochés de telle sorte qu'il puissent être lus des deux côtés ; ou encore dans le cas des livres de W. $G$. Sebald que nous exposons avec sa bibliothèque pour 
Vue de l'exposition Ernst Jünger, travailleur au bord de l'abîme

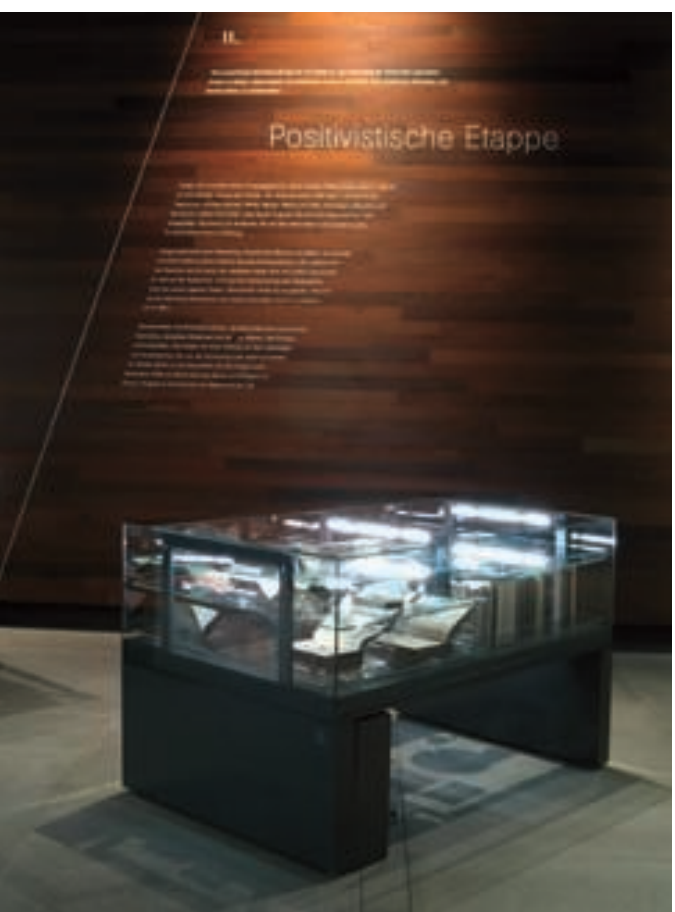

Vue générale de l'exposition Ernst Jünger, travailleur au bord de l'abîme

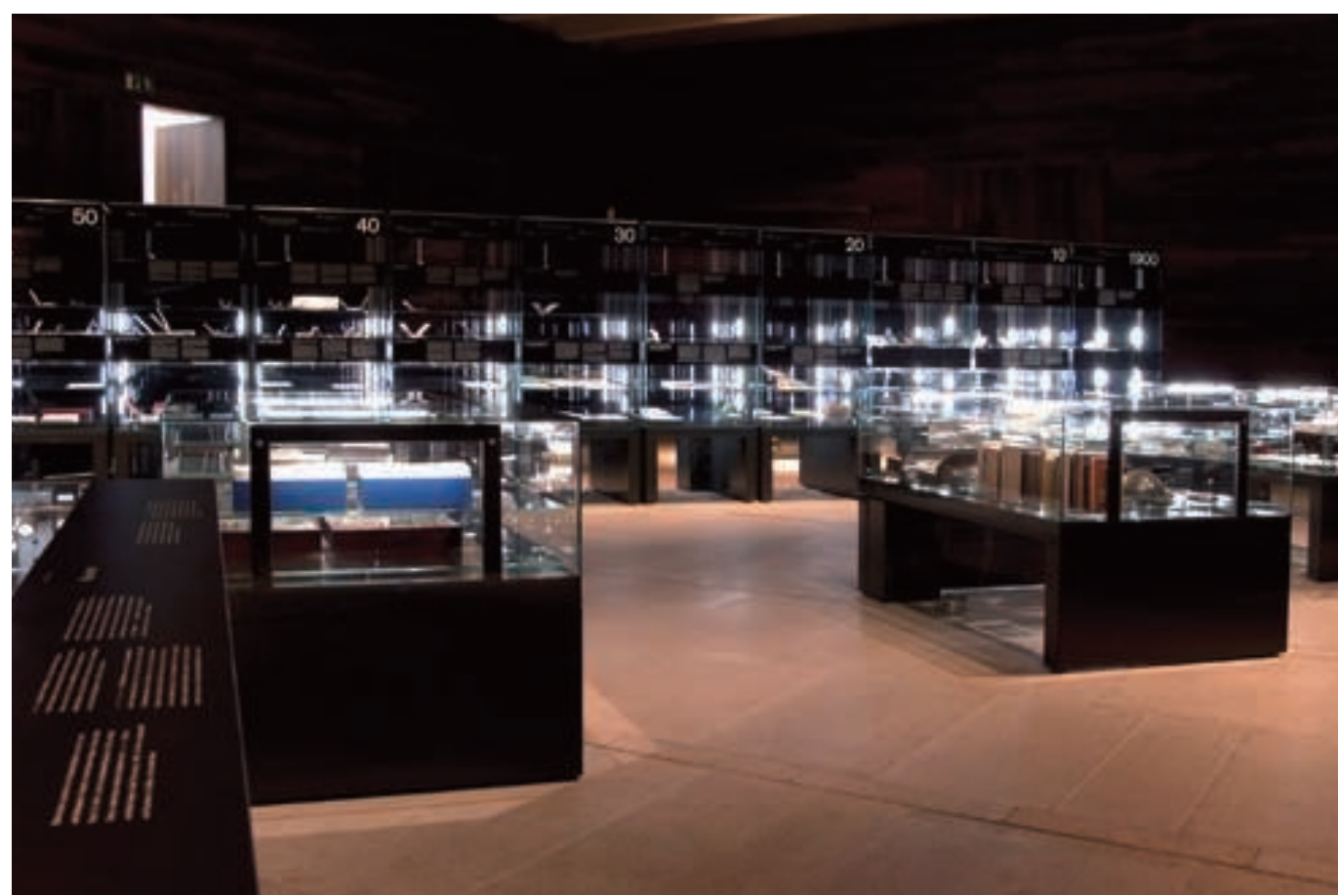

en souligner l'intertextualité). Parfois les documents doivent être transcrits car il est impossible de les déchiffrer ; parfois ils doivent être replacés dans un contexte plus large. Dans d'autres cas, on passe par le biais des objets, comme dans l'exposition conçue en 2009 pour l'année Schiller, dans laquelle nous avons utilisé les reliques corporelles et vestimentaires de Schiller comme autant de mots-clés pour montrer les particularités de son écriture qui réunit le corps et l'âme, le mouvement et la pensée, la réalité et la poésie, ou bien encore lors de l'exposition Ordre. Une histoire sans fin, consacrée aux relations complexes qu'entretiennent les écrivains avec le concept d'ordre. Les textes explicatifs des expositions n'évaluent pas les objets, ne racontent pas une histoire qui pourrait aussi être racontée sans leur présence ni ne se targuent d'objectivité ou de valorisation (ce qui est présenté et pourquoi c'est important, ou bon, ou mauvais). Les textes doivent conduire un peu le visiteur, l'inciter à regarder par lui-même et attiser la passion sans laquelle on ne pourrait pas réaliser une exposition d'archives : la passion de découvrir dans de petits objets insignifiants quelque chose qui nous permet de mieux les comprendre et de mieux comprendre la littérature. Ils nous conduisent sur les chemins d'une connaissance que, sans eux, nous n'aurions pas acquise et qui nous transforme et nous transporte. Dans le meilleur des cas, ils permettent des épiphanies au milieu du quotidien. Nous avons utilisé, dans l'exposition consacrée à Jünger, différentes sortes de textes. La vitrine des écrits est accompagnée de textes longs qui aident à voir et à lire, décrivent et transcrivent. Les vitrines thématiques sont accompagnées de textes correspondants qui s'intéressent à la langue métaphorique de Jünger et soulignent, sur un mode à la fois poétique et parlant, les mouvements de sa pensée, la façon dont elle passe d'un niveau à un autre.

\section{Au-delà de l'exposition}

L'exposition est conçue pour tous les visiteurs, ceux pour qui Jünger n'évoque rien, ceux qui n'ont de l'écrivain qu'une idée vague ou partiale comme ceux qui le connaissent bien. On peut la traverser en dix minutes en se contentant de regarder mais on peut aussi y passer trois heures et beaucoup lire. Dans les deux cas, on en repartira avec au moins une impression : celle de quelqu'un qui a passé sa 
longue vie à écrire et n'a cessé de retravailler ses textes, avec le temps ou contre le temps. Le visiteur est libre des sentiments que cela lui inspire, qu'il juge Jünger passionnant ou ennuyeux, que fasciné, il ait envie de poursuivre sa découverte ou qu'il s'arrête et ressorte effrayé. Cette exposition qui aiguise notre regard sur l'œuvre de Jünger en partant de son mode de production n'apportera rien à celui qui aime s'identifier à un auteur et cherche dans son œuvre un message concret. L'exposition montre de Jünger l'image la plus ennuyeuse qui soit, celle de l'écrivain infatigable travailleur, qui écrit et réécrit sans cesse ses textes. C'est ce qui nous a paru le plus adapté, en tout cas à ce lieu, et ce qui, loin de nous en détourner, nous conduit vers son œuvre et son mélange particulier de récits et de philosophie de la vie. Peutêtre est-ce ici que réside la nouveauté. Nous sommes partis des fondamentaux de la littérature - le travail sur et avec la langue - sans craindre de déconcerter les visiteurs. Jünger est un auteur lointain, non pas au sens où son expérience nous serait étrangère (la guerre et l'enthousiasme qu'elle provoque, les fantasmes masculins et la passion de la chasse, l'énergie atomique et les expériences des drogues, le travail et la technique, les voyages lointains, les marginaux et les rôdeurs font toujours partie de notre réalité), mais parce que son style, proche de la modernité née autour de 1900, nous apparaît aujourd'hui difficile et déconcertant. On peut citer l'absence de narration à la première personne, les forces magiques, oniriques, voire théologiques prêtées à la nature, le style froid et flegmatique, l'accumulation de métaphores, tout ce que Gottfried Benn a qualifié d'" enflé " parce que l'auteur ne disparaît jamais vraiment de ce style instantané (que Benn qualifie de "Sekunden-Stil "); sans oublier l'absence d'action qui tienne en haleine, l'utilisation d'aphorismes et de rêves, l'ancrage de cette écriture dans des expériences historiques comme la Première Guerre mondiale, les réflexions philosophiques, l'amour des analogies et de la mythologie et la volonté de mythifier le sujet. On peut penser, si l'on se considère moralement supérieur à Jünger, et à la lumière des gouffres historiques qui se sont creusés autour de lui (la cruauté des deux guerres mondiales et de l'holocauste), que ces procédés stylistiques sont cyniques et de mauvais goût. Nous nous sommes contentés de montrer la différence entre ces procédés et la réalité historique et de, littéralement, dé-construire cette œuvre. "Ici, chaque classique est examiné jusqu'à l'os comme lors d'une radiographie. Il ne reste pas grand-chose de la grandeur marmoréenne. Il n'en a pas été autrement avec Jünger. L'exposition Ernst Jünger, travailleur au bord de l'abîme donne la mesure de ce qu'une exposition littéraire peut apporter à la compréhension d'une œuvre " (Die Zeit, Iljoma Mangold).

\section{Heike Gfrereis \\ (traduction Aude Therstappen)}

\section{RÉFÉRENCES BIBLIOGRAPHIQUES :}

Un catalogue de l'exposition a été publié avec des articles de Stephan Schlak, Heike Gfrereis (avec la collaboration de Sonja Lehmann et Ellen Strittmatter), Detlev Schöttker et des entretiens de Stephan Schlak avec Helmut Lethen et Karl Heinz Bohrer (Ernst Jünger. Arbeiter am Abgrund, Marbach, 2010, 284 pages, ill. en couleurs, broché, $26 €$, ISBN 978-3-937384-69-6).

Le commissariat général de l'exposition a été assuré par Heike Gfrereis et Ellen Strittmatter, la scénographie et la conception graphique de l'exposition ont été réalisées par Diethard Keppler, Markus Wichmann et le bureau d'architectes stuttgartois Space4.

Vous trouverez des informations sur le programme de manifestations autour de l'exposition, sur le catalogue et les deux musées des Archives littéraires allemandes à l'adresse suivante : www.dla-marbach.de/museum.

\section{SUR LES EXPOSITIONS DES ARCHIVES DE MARBACH :}

Anne Bohnenkamp-Renken et Sonja Vandenrath (dir.), Wort-Räume, Zeichen-Wechsel, Augen-Poesie. Zur Theorie und Praxis von Literaturausstellungen, Göttingen, 2010

Heike Gfrereis, Didaktik des Schweigens. Das Literaturmuseum der Moderne, in Der Deutschunterricht, Heft 2, 2009 